\title{
Response of the antioxidant system of two varieties of potatoes to salt stress
}

\author{
O.K. Murgan*, L.V. Kolomeichuk, I.S. Kovtun, M.V. Efimova
}

Tomsk State University, Tomsk, Russia

DOI 10.18699/ICG-PlantGen2019-31

(c) Autors, 2019

*e-mail: reborn_rinni@mail.ru

\begin{abstract}
The mechanisms of potato (Solanum tuberosum L.) plants' tolerance to chloride salinity were investigated in the early-season cv. 'Zhukovsky' and the mid-season cv. 'Lugovskoy'. At an age of 6 weeks, the plants were exposed to $100 \mathrm{mM} \mathrm{NaCl}$ stress for 7 days. Plant response to salt stress was estimated by physiological parameters (content of proline and activity of antioxidant enzymes) and the gene expression level for enzymatic and nonenzymatic antioxidant system. Early- and mid-ripening varieties of potato, in response to potato salinity, have differences in activity of guaiacol-dependent peroxidase, levels of gene expression of ascorbate peroxidase, and also accumulate toxic ions in leaves and roots, which has for the first time been shown in this study.
\end{abstract}

Key words: Solanum tuberosum L.; salt tolerance; antioxidant system.

\section{Introduction}

The growing season duration for potato plants is one of the key criteria for their regionalization in a certain climate zone. For the Siberian region characterized by a short summer season, early-maturing varieties are most preferred. However, the increase in saline areas sets priority on salt tolerance varieties. Salinization negatively affects the implementation of many physiological processes in plants, largely due to the generation of reactive oxygen species and the development of oxidative stress, which leads to disruption of the photosynthetic apparatus, a decrease in the intensity of photosynthesis and, as a consequence, a decrease in the productivity of plants; induction of aging or premature death of the plant. In response to the oxidative stress progress and metabolic disturbances, the plant responds by activating the cellular antioxidant system, which includes antioxidant enzymes (catalases, peroxidases, superoxide dismutases (SOD), etc.) and low-molecular-weight organic compounds with antioxidant properties (proline, phenolic compounds, carotenoids). In this article, the functioning of the antioxidant system for early- and mid-season varieties potato plants based on the proline content and antioxidant enzymes activity was studied. Also, gene expression levels of the enzymatic (APX1, APX3) and the non-enzymatic (P5CS1, $\mathrm{P} 5 \mathrm{CR}, \mathrm{PDH}$ ) plant protection systems under chloride salinity were discussed.

\section{Materials and methods}

The study was carried out on Solanum tuberosum L. plants of the early-season cv. 'Zhukovsky' and the mid-season cv. 'Lugovskoy'. Virus-free in vitro potato regenerants were cultivated for 21 days on the half-strength Murashige and Skoog $(1 / 2 \mathrm{MS})$ agar medium, after that the roots of the plants were washed from the agar medium and grown for three weeks on a $1 / 2$ MS liquid medium. To model salt stress, the plants were transferred for 7 days to $1 / 2$ MS with the addition of $100 \mathrm{mM}$ $\mathrm{NaCl}$. Half-strength MS medium without $\mathrm{NaCl}$ was used as a reference. Evaluation of physiological characteristics was performed according to the methods described previously (Efimova et al., 2018). Total RNA from leaf tissue was iso- lated using Manickavelu`s method (Manickavelu et al., 2007). Cyclofilin and elongation factor 1- $\alpha$ were used as reference genes. Levels of gene transcript contents were determined by real-time PCR (SYBRGreen I dye) using the LightCycler 96 System (Roche, Switzerland).

Our experiments were carried out in three biological replicates. The figures present the mean values and their standard errors (SE). The means were compared using ANOVA on Ranks (Kruskal-Wallis criterion).

\section{Results and discussion}

Plant adaptation to a disturbance of water status and a toxic effect of salinity mostly depends on compatible osmolytes acting as chemical chaperones. We revealed that accumulation of the universal compatible osmolyte proline in potato plants is species specific. Figure $1 a$ shows that the content of this osmoprotector in the leaves 'Zhukovsky' is two times higher than in 'Lugovskoy'.

In response to salinity (100 $\mathrm{MM} \mathrm{NaCl})$, plants showed a six- and sevenfold increase in proline content in the leaves of the early- and the mid-season variety, respectively.

To determine the mechanisms of proline accumulation in the leaves of plants, the expression of genes for proline synthesis (P5CS1 and P5CR) and proline degradation (PDH) was analyzed (Figure 1, $b, c, d$ ). In the absence of a stressor, the level of transcripts for proline synthesis genes was the same for both varieties, while the level of PDH gene expression in 'Zhukovsky' was two times higher than in 'Lugovskoy'. The variability of proline metabolism transcription genes under salinity was noted. 'Zhukovsky' plants responded to the stressor by inhibiting proline synthesis gene expression, while in 'Lugovskoy', an increase in P5CS1 and PDH expression was observed (see Figure 1, $b, c, d$ ).

To reduce the negative effects of oxidative stress triggered by $\mathrm{NaCl}$, the enzymatic antioxidant defense systems in plants can be activated. The superoxide radical is known to be a primary product of one-electron reduction of molecular oxygen and a precursor of other, frequently more aggressive ROS. Therefore, superoxide dismutase-catalyzed conversion of 

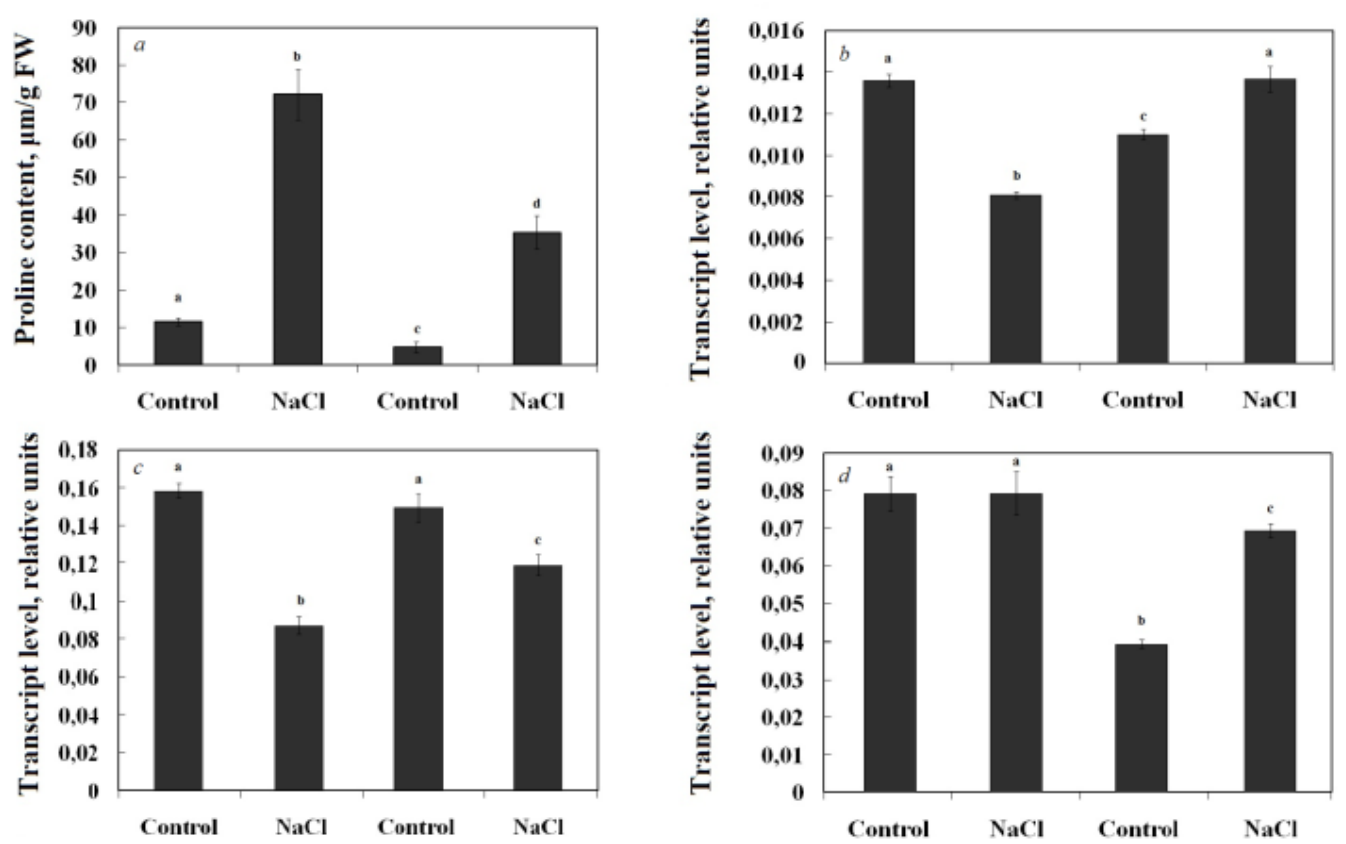

Zhukovsky

Lugovskoy

Zhukovsky

Lugovskoy

Figure 1. Effect of $100 \mathrm{mM} \mathrm{NaCl}$ on proline content (a) and transcript levels of P5CS1 (b), P5CR (c) and PDH (d) genes in S. tuberosum leaves. Letters indicate significant differences $(p<0.05)$ between the experiment variants.
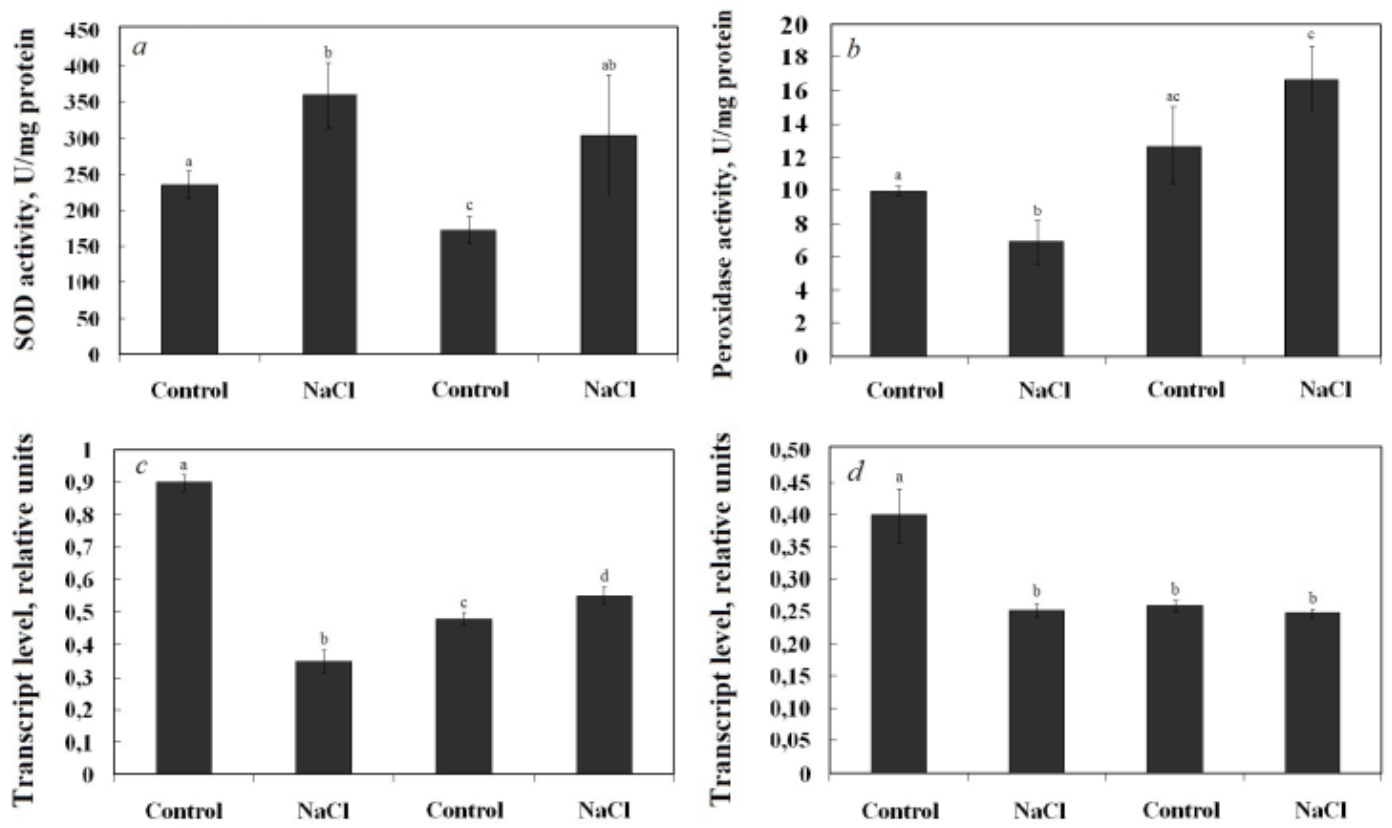

Zhukovsky

Lugovskoy

Zhukovsky

Lugovskoy

Figure 2. Effect of $100 \mathrm{mM} \mathrm{NaCl}$ on superoxide dismutase (a) and peroxidase activity (b), transcript levels of APX1 (c) and APX3 $(d)$ in S. tuberosum leaves. Letters indicate significant differences $(p<0.05)$ between the experiment variants. 
$\mathrm{O}_{2}^{-}$into $\mathrm{H}_{2} \mathrm{O}_{2}$ plays a key role in plant cell protection against oxidative destruction (Kartashov et al., 2008).

Guaiacol-dependent peroxidase catalyses the conversion of hydrogen peroxide into water. The activity of superoxide dismutase and peroxidase in the leaves of plants of the earlyand the mid-season potato variety was evaluated (Figure 2, $a, b)$. It has been shown that, under optimal conditions, 'Zhukovsky' had higher SOD activity and lower peroxidase activity compared with 'Lugovskoy'. During salinity, SOD and peroxidase activity increased 1.8 and 1.3 times, respectively, in 'Lugovskoy' leaves; while the plants of the earlyripening variety responded to stress by a 1.5 -fold increase in SOD activity and a 1.4-fold decrease in peroxidase activity (see Figure 2, $a, b$ ).

An important role in the regulation of the level of hydrogen peroxide in plant cells is played by ascorbate peroxidase (APX), which catalyzes the conversion of hydrogen peroxide into water with the participation of ascorbic acid. Ascorbic acid is dehydrolized to dehydroascorbate and acts as a substrate of reactions involving guaiacol-dependent peroxidase (Yang et al., 2018). We studied the expression level of the cytosolic ascorbate peroxidase gene APX1 and the peroxisomal ascorbate peroxidase gene APX3 (see Figure 2, c, d). The transcript level of the two APX genes was 1.5-1.9 times higher in 'Zhukovsky' than 'Lugovskoy' leaves. Transcript levels of the APX1 and APX3 genes in 'Zhukovsky' in stress condition decreased 1.6-2.6 times compared to the control variant, while 'Lugovskoy' plants kept the transcript levels for both genes at the control level (see Figure 2,c,d). Assay of the weight content of sodium and chlorine ions during salinization by the method of energy dispersive analysis on the Quanta 200 3D EDAX scanning electron microscope (Netherlands) showed that the mid-ripening potato variety 'Lugovskoy' response to $\mathrm{NaCl}$ was characterized by a smaller accumulation of toxic sodium and chlorine ions in the leaves and roots than the 'Zhukovsky' response, which indicates a higher salt tolerance of the mid-ripening variety (data not shown).

\section{Conclusions}

For the first time, the causes of potato plant resistance to $\mathrm{NaCl}$ action for the varieties analyzed were identified and a detailed comparison of the functioning of the protective systems of potato plants depending on the maturation period was made. We have shown that, in the case of chloride saliniza- tion, the early- and middle-ripening varieties regulated the functioning of the antioxidant system of plants by different ways. Thus, the early-ripening variety 'Zhukovsky' under the action of $\mathrm{NaCl}$ accumulates proline in the leaves and increases the activity of superoxide dismutase; at the same time, peroxidase activity decreases and the levels of the APX1 and APX3 genes are reduced. 'Lugovskoy' plants respond to the action of the stressor by a greater accumulation of proline, an increase in SOD and guaiacol-dependent peroxidase activities. It is considered that the activity of guaiacol-dependent peroxidase was to a much greater degree correlated with the plant capability of adaptation than proline accumulation and SOD activity (Kartashov et al., 2008). These data indicate a higher antioxidant activity for 'Lugovskoy' plants. In addition, plants of this variety accumulate less toxic ions of sodium and chlorine in the leaves and roots under stress condition than 'Zhukovsky' plants. The high salt tolerance of 'Lugovskoy' plants can be caused not only by increased antioxidant activity, but also by the reduced salt-absorbing capacity of root cells, as well as by controlled selective transport of ions to the aboveground organs.

\section{References}

Efimova M.V., Kolomeichuk L.V., Boyko E.V., Malofii M.K., Vidershpun A.N., Plusnin I.N., Golovatskaya, I.F., Murgan O.K., Kuznetsov V.V. Physiological Mechanisms of Solanum tuberosum L. Plants' Tolerance to Chloride Salinity. Russian J Plant Physiol. 2018;65(3):394-403. DOI 10.1134/S1021443718030020.

Manickavelu A. et al. Colloids and Surfaces B: Biointerfaces. 2007; $54: 254-258$

Nicot N., Hausman J.F., Hoffmann L., Evers D. Housekeeping gene selection for real-time RT-PCR normalization in potato during biotic and abiotic stress. J Experimental Bot. 2005;56(42):2907-2914. https://doi.org/10.1093/jxb/eri285.

Kartashov A.V., Radyukina N.L., Ivanov Yu.V., Pashkovskii P.P., Shevyakova N.I., Kuznetsov V.V. Role of antioxidant systems in wild plant adaptation to salt stress. Russian Journal of Plant Physiology. 2008;55(4):463-468. DOI 10.1134/S1021443708040055.

Yang Y., Guo Y. Elucidating the molecular mechanisms mediating plant salt-stress responses. New Phytol. 2018;217(2):523-539.DOI 10.1111/nph.14920

Acknowledgements. The work is supported by the granting program of Russian Foundation for Basic Research project No. 17-54-61017Egypt_a.

Conflict of interest. The authors declare no conflict of interest. 